\title{
Il modello della fessura coesiva in trazione e compressione per la valutazione della duttilità degli elementi strutturali in calcestruzzo armato
}

\author{
Alberto Carpinteri, Mauro Corrado, Giuseppe Mancini, Marco Paggi \\ Politecnico di Torino - Dipartimento di Ingegneria Strutturale e Geotecnica, corso Duca degli Abruzzi 24, 10129, Torino, Italy
}

RiAssunTO. Il problema della valutazione della duttilità degli elementi in calcestruzzo armato soggetti a flessione o presso-flessione è stato largamente studiato negli ultimi decenni, sia da un punto di vista sperimentale che analitico. Data l'influenza di numerosi parametri di progetto sulla duttilità, tuttavia, è difficile sviluppare un modello in grado di descrivere completamente la risposta meccanica di elementi strutturali, tenendo conto di tutti gli effetti dovuti alla non-linearità dei materiali. Nel passato, in particolare, si è studiato in maniera approfondita l'effetto della classe di duttilità dell'acciaio, mentre il ruolo degli effetti di scala, evidenziato da più campagne sperimentali, non è stato ancora del tutto chiarito. Una delle ragioni principali è l'inadeguatezza dei modelli tradizionali, basati su leggi costitutive tra tensioni e deformazioni. Nel presente lavoro, si propone un nuovo modello basato sul concetto della localizzazione delle deformazioni, capace di descrivere la propagazione della fessura e l'avanzamento del crushing durante il processo di carico. In tale contesto, il comportamento non-lineare del calcestruzzo in compressione è modellato attraverso l'Overlapping Crack Model, modello analogo a quello coesivo valido per la trazione, che descrive la localizzazione delle deformazioni dovuta al danneggiamento del calcestruzzo mediante una compenetrazione del materiale. Con questo nuovo algoritmo è possibile cogliere l'effettiva risposta flessionale di elementi strutturali in calcestruzzo armato al variare della percentuale di armatura e della scala dimensionale. Applicazioni numeriche riguardano l'analisi della risposta post-picco di provini in calcestruzzo soggetti a compressione e la valutazione delle rotazioni plastiche di travi in calcestruzzo armato soggette a flessione su tre punti. Si propone infine un ampio confronto con i risultati di prove sperimentali, con lo scopo di dimostrare la validità del nuovo approccio.

ABSTRACT. The problem of assessing the ductility of reinforced concrete (RC) structural elements in bending or under the action of eccentric forces has been largely investigated from both the experimental and the analytical point of view during the last decades. Since the development of ductility is influenced by several design parameters, it is difficult to develop a predictive model able to fully describe the mechanical behaviour of the structural element. In particular, the role of the size-scale effect, which has been evidenced by some experimental tests, is not yet completely understood. One of the main reasons is the inadequacy of the traditional models based on ad hoc stress-strain constitutive laws. In the present contribution, a new model based on the concept of strain localization is proposed, which is able to describe both cracking and crushing growths during the loading process. In particular, the nonlinear behaviour of concrete in compression is modelled by the Overlapping Crack Model, which describes the strain localization due to crushing by means of a material compenetration. With this numerical algorithm in hand, it is possible to effectively capture the flexural behaviour of RC structural elements by varying the reinforcement percentage and/or the structural size. Numerical applications regard the analysis of the post-peak nonlinear response of concrete specimens subjected to eccentric compression tests and the evaluation of the plastic rotation of RC beams under three-point bending. An extensive comparison with experimental results is also proposed, fully demonstrating the effectiveness of the proposed approach. 
Parole Chiave. Calcestruzzo armato, Rotazioni plastiche, Effetti di scala, Crushing, Modello coesivo, Metodo degli Elementi Finiti.

\section{INTRODUZIONE}

$\mathrm{I}$ l comportamento del calcestruzzo in compressione, e in particolar modo la resistenza a compressione e la risposta meccanica nella fase di post-picco, hanno un ruolo predominante nel progetto di strutture in calcestruzzo semplice e armato. Il progetto di elementi strutturali, infatti, è basato sul confronto tra una caratteristica sollecitante e la corrispondente resistenza, valutata sulla base della resistenza propria del materiale e del meccanismo di collasso. Il comportamento del materiale nella fase di post-picco è invece fondamentale per una corretta valutazione della duttilità della struttura, come ad esempio nella valutazione della deformazione assiale ultima di pilastri o della capacità di rotazione plastica di travi in calcestruzzo armato. In questo contesto, gli effetti della dimensione strutturale giocano un ruolo importante, dovuto al fatto che i parametri caratteristici del calcestruzzo vengono valutati su provini alla scala del laboratorio, alquanto diversa dalla dimensione delle strutture reali. Da qui la necessità di sviluppare modelli analitici o numerici che permettano di estrapolare correttamente i risultati delle prove sperimentali alle grandi scale.

L'interesse per la duttilità delle strutture risale all'inizio del secolo scorso, con il diffondersi del calcolo plastico come metodo di valutazione del carico ultimo di una struttura [1]. In questo contesto, tale proprietà è fondamentale per permettere la ridistribuzione dei momenti flettenti all'interno di strutture iperstatiche. La duttilità può essere opportunamente valutata attraverso la capacità di rotazione delle cerniere plastiche che si sviluppano nelle sezioni più sollecitate. A tal proposito, due possibili definizioni di rotazione plastica sono state proposte in letteratura. In base al Model Code 90 [2], tale rotazione è definita come la differenza tra la rotazione corrispondente al massimo momento resistente e quella relativa allo snervamento dell'acciaio, come rappresentato schematicamente da $\vartheta_{P L}^{(1)}$ in Fig. 1 . Al fine di considerare il contributo di duttilità sviluppato oltre il carico massimo, Hillerborg [3] e Pecce [4] hanno proposto una misura alternativa della rotazione plastica, come differenza tra la rotazione oltre la quale si ha un rapido decremento del momento resistente e la rotazione di snervamento dell'acciaio $\left(\vartheta_{P L}^{(2)}\right.$ in Fig. 1). Queste misure sono entrambe proporzionali alla duttilità delle travi in calcestruzzo armato.

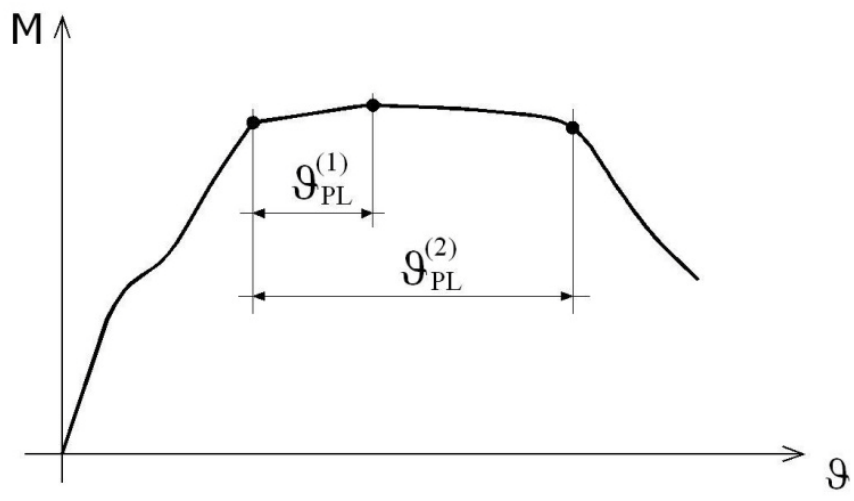

Figura 1: Differenti definizioni di rotazione plastica nel diagramma momento-rotazione della sezione più sollecitata.

Data la complessità del fenomeno di formazione e di sviluppo delle cerniere plastiche, i primi studi sulla capacità rotazionale derivano dalle prove sperimentali condotte negli anni '60 del secolo scorso, coordinate dalla "Indeterminate Structures Commission" del CEB [5], presieduta dal Prof. Baker. Sulla base dei risultati sperimentali ottenuti, pubblicati nel 1967 [6], si decise di descrivere la rotazione plastica in funzione della profondità relativa dell'asse neutro, $x / d$. Di conseguenza, fu proposta la seguente relazione iperbolica, che costituisce la curva approssimante il frattile 5\% dei risultati:

$$
\vartheta_{P L}=0.004 \frac{d}{x}
$$


Tale espressione fu assunta da alcune normative dell'epoca, come ad esempio il Model Code 78, per risolvere il problema della valutazione delle rotazioni plastiche ammissibili ai fini progettuali.

Un secondo importante contributo fu dato dalla ricerca condotta agli inizi degli anni '80 presso l'Università di Stoccarda dal gruppo coordinato dal Prof. Eligehausen [7]. In tale occasione fu sviluppato un modello analitico per l'analisi delle cerniere plastiche considerando l'esistenza di due tipi di collasso: lato acciaio e lato calcestruzzo. Le curve proposte sono confrontate in Fig. 2 con la legge espressa dall'Eq. (1). Due aspetti fondamentali devono essere evidenziati: la presenza di un ramo crescente per piccoli valori di $x / d$ dovuto alla rottura lato acciaio e la presenza di due curve relative ad acciai aventi caratteristiche di duttilità differenti. Tali curve furono in seguito adottate dal Model Code 90. Successivamente altri modelli furono sviluppati, enfatizzando uno o più particolari aspetti del problema [8-10].

La più recente indicazione normativa è quella presente nell'ultima versione dell'Eurocodice 2 Parte 2 [11], riportata in Fig. 3. Le linee tratteggiate si riferiscono ad acciai ad alta duttilità (Classe C), mentre quelle a tratto pieno si riferiscono ad acciai a normale duttilità (Classe B).

Dall'analisi delle indicazioni delle normative si può evidenziare come l'effetto della scala sulla capacità rotazionale sia stato trascurato, sebbene numerose campagne sperimentali abbiano evidenziato una forte influenza di tale fenomeno [12-15].

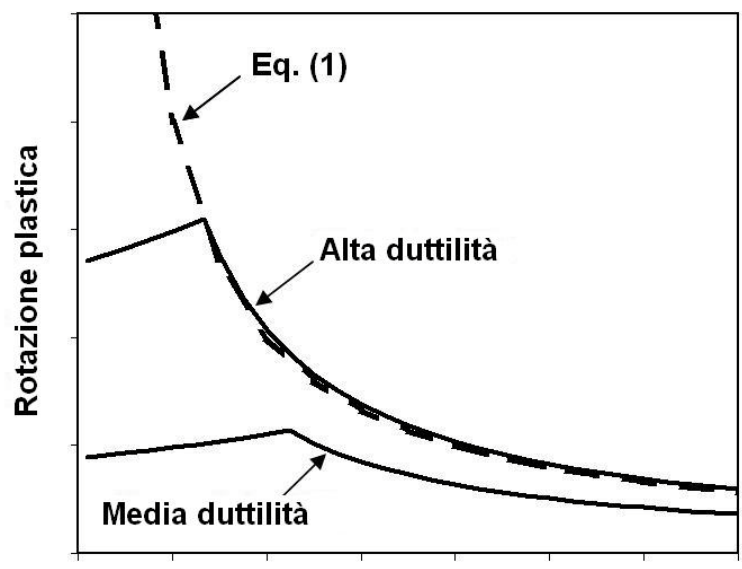

Profondità relativa dell'asse neutro $(x / d)$

Figura 2: Evoluzione delle formule di progetto per il calcolo della rotazione plastica ammissibile.

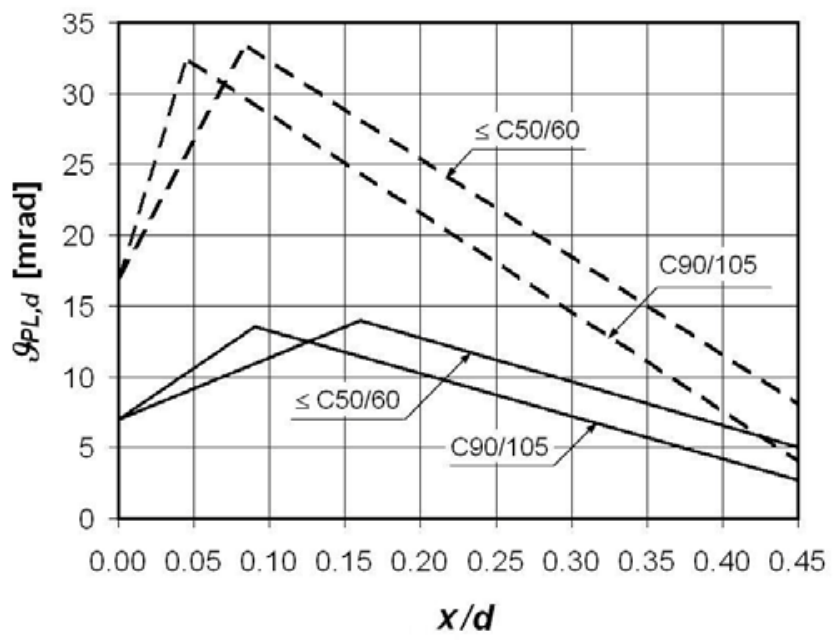

Figura 3: Legami tra rotazione plastica ammissibile e profondità relativa dell'asse neutro in base all'Eurocodice 2.

Come intuito da Hillerborg fin dal 1990 [3], la causa degli effetti di scala, anche per quanto riguarda la capacità di rotazione, risiede nella localizzazione delle deformazioni, sia in trazione che in compressione. Hillerborg fu il primo ad introdurre tale concetto, decisamente più intuitivo nel caso della trazione, anche in compressione. In base al suo approccio, una volta raggiunta la resistenza a compressione, si verifica una localizzazione delle deformazioni all'interno di una zona della trave avente una lunghezza pari alla profondità della zona compressa. Bažant [16] ha proposto un modello 
analogo, nel quale la zona di localizzazione è definita come lunghezza caratteristica del materiale. Tale modello permette di affrontare il problema degli effetti di scala, ma di fatto non permette di ottenere una legge costitutiva propria del materiale, essendo la lunghezza di localizzazione proporzionale alla dimensione del provino. Inoltre, in entrambi i modelli, la lunghezza di localizzazione non è definita sulla base di valutazioni teoriche ma da un best-fitting di risultati sperimentali. D'altro canto, la localizzazione delle deformazioni in compressione è stata osservata in numerose campagne sperimentali [17-22].

Nel presente lavoro, il comportamento del calcestruzzo in compressione viene descritto mediante un nuovo modello, denominato Overlapping Crack Model, nel quale il processo di collasso del calcestruzzo in compressione è descritto in modo analogo a quello in trazione. In trazione la localizzazione delle deformazioni è rappresentata dall'apertura della fessura, mentre in compressione è descritta da una compenetrazione del materiale, come mostrato in Fig. 4. Questi due modelli elementari vengono poi coniugati in un modello numerico più complesso sviluppato per descrivere il comportamento delle cerniere plastiche in elementi in calcestruzzo armato. Tale modello sarà successivamente validato mediante confronto con i risultati di prove sperimentali condotte su travi in calcestruzzo armato da Bosco e Debernardi [14]. Infine, sarà proposto un confronto tra i risultati del modello e le prescrizioni dell'Eurocodice 2 riguardanti la capacità rotazionale di travi in calcestruzzo armato.

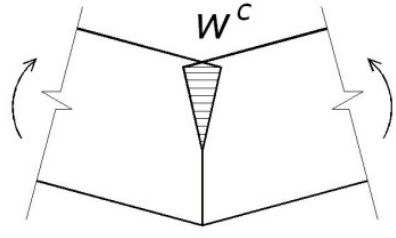

(a)

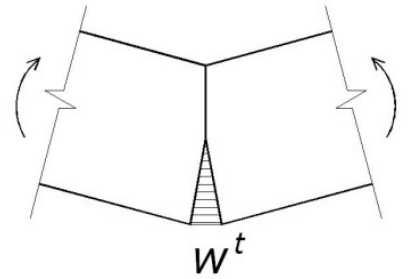

(b)

Figura 4: Compenetrazione in compressione (a); analoga alla fessura coesiva in trazione (b).

\section{ANALISI NUMERICA}

I n questa sezione si propone un nuovo modello basato sui concetti della Meccanica della Frattura per la valutazione della capacità di rotazione plastica di travi in calcestruzzo armato soggette a flessione. L'analisi è condotta su un concio di trave avente lunghezza pari all'altezza, soggetto a momento flettente costante. Tale elemento è considerato rappresentativo della zona di formazione della cerniera plastica, coerentemente con quanto suggerito dall'Eurocodice 2 . Si assume, inoltre, che i processi di frattura in trazione e di crushing in compressione siano localizzati nella sezione di mezzeria, mentre la parte restante abbia un comportamento elastico. Ciò implica che sia considerata un'unica fessura equivalente, anziché una fessurazione diffusa.

La distribuzione delle tensioni nella sezione di mezzeria è elastica-lineare fino al raggiungimento della resistenza a trazione in corrispondenza del lembo inferiore. Quando tale limite è raggiunto, una fessura coesiva si propaga dall'intradosso verso l'estradosso del concio. Al di fuori della fessura il materiale ha comportamento elastico. In questa fase il momento esterno aumenta, la fessura si estende e la tensione di compressione al lembo superiore aumenta fino a raggiungere la resistenza a compressione. A questo punto inizia il danneggiamento a compressione del calcestruzzo con la conseguente localizzazione dell'energia dissipata, che viene descritta mediante la compenetrazione del materiale. Più grande è la compenetrazione (overlapping), più piccoli sono gli sforzi di compressione trasmessi dai due elementi.

\section{Il modello coesivo per descrivere la frattura del calcestruqzo in trazione}

In base al modello coesivo [23, 24], la legge costitutiva utilizzata per il materiale non danneggiato è una relazione $\sigma-\varepsilon$ lineare-elastica fino al raggiungimento della resistenza a trazione. Nella zona di processo, il materiale danneggiato è ancora in grado di trasmettere sforzi attraverso le facce della fessura. Tali sforzi sono inversamente proporzionali all'apertura della fessura, $w^{t}$, secondo la seguente espressione:

$$
\sigma_{\mathrm{t}}=\sigma_{\mathrm{t}, \mathrm{u}}\left(1-\frac{w^{\mathrm{t}}}{w_{\mathrm{cr}}^{\mathrm{t}}}\right)
$$


dove: $w^{t}$ è l'apertura della fessura, $w_{c r}^{t}$ è il valore critico dall'apertura della fessura oltre il quale si annullano gli sforzi trasmessi e $\sigma_{\mathrm{t}, \mathrm{u}}$ è la resistenza a trazione.

L'area sottesa dalla curva tensione-spostamento rappresenta l'energia di frattura, $G_{\mathrm{F}}^{\mathrm{t}}$.

\section{Il modello di overlapping per descrivere la rottura del calcestruz:o in compressione}

In ambito strutturale, le leggi costitutive maggiormente adottate per il calcestruzzo in compressione descrivono il comportamento del materiale in termini di tensione in funzione della deformazione (legge elasto-plastica, parabolarettangolo, parabola di Sargin, ecc.). Questo approccio, che implica una dissipazione di energia all'interno dell'intero volume, non permette di descrivere correttamente il comportamento meccanico al variare della dimensione strutturale. Al contrario, gli effetti della scala sono dovuti alla localizzazione delle deformazioni all'interno di una banda di danneggiamento trasversale o inclinata [17-19] e ad una conseguente localizzazione dell'energia dissipata nella fase di postpicco. A tal proposito, è stato evidenziato come l'energia dissipata per unità di volume diminuisca all'aumentare della scala, mentre, la stessa energia, rapportata all'area della sezione trasversale, possa essere considerata costante [20-22].

Alcuni Autori $[17,18]$ hanno sperimentalmente evidenziato che, da un punto di vista globale, senza considerare in dettaglio il meccanismo di collasso del provino, lo schiacciamento localizzato può essere considerato come una caratteristica del materiale nella fase di post-picco, analogamente a quanto avviene per l'apertura della fessura in trazione. Sulla base di tale osservazione è possibile introdurre un modello più generale, per cui la deformazione irreversibile dovuta al fenomeno di danneggiamento è descritta da una compenetrazione fittizia, mentre la parte restante di provino è soggetta ad uno scarico elastico, come proposto da Carpinteri et al. [25]. Di conseguenza viene introdotta una doppia legge costitutiva: un legame $\sigma-\varepsilon$ fino al raggiungimento della resistenza a compressione (Fig. 5a) e un legame $\sigma-w$ (compenetrazione) descrivente la rottura a compressione del calcestruzzo (Fig. 5b). Quest'ultima relazione descrive il modo in cui la tensione nel materiale danneggiato diminuisce dal valore massimo fino a zero all'aumentare della compenetrazione da zero fino al valore critico $w_{\mathrm{cr}}$.

È importante notare che la compenetrazione è una quantità integrata che permette di caratterizzare il comportamento strutturale senza la necessità di modellare nello specifico il reale meccanismo di rottura, che può variare dalla frantumazione, alla rottura diagonale per taglio, allo splitting, variando la scala e la snellezza del provino compresso.

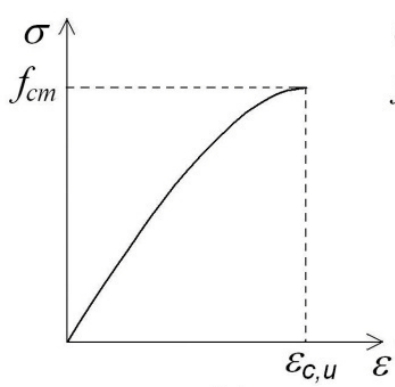

(a)

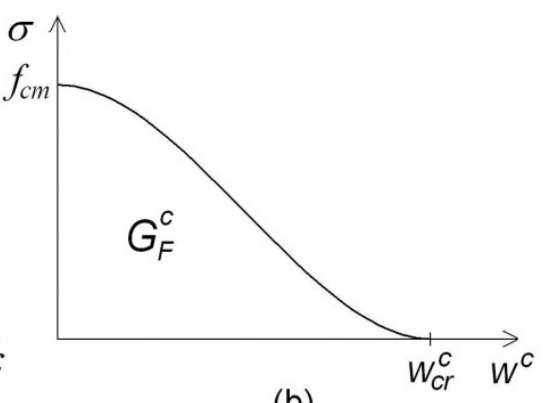

(b)

Figura 5: Modello di Overlapping: legame tensione-deformazione (a); legame tensione-compenetrazione (b).

L'area sottesa dalla curva $\sigma-w$ riportata in Fig. 5b rappresenta l'energia di crushing, $G_{\mathrm{F}}^{\mathrm{c}}$, definita come energia dissipata per unità di superficie. Tale parametro è una proprietà del materiale dal momento che non è affetta dalla scala strutturale. La formulazione empirica utilizzata nel presente lavoro per determinarne il valore è stata proposta da Suzuki et al. [19], ed è basata su risultati di prove a compressione uniassiale condotte su provini in calcestruzzo semplice e armato trasversalmente:

$$
\frac{G_{\mathrm{F}}^{\mathrm{c}}}{\sigma_{\mathrm{c}, 0}}=\frac{G_{\mathrm{F}, 0}^{\mathrm{c}}}{\sigma_{\mathrm{c}, 0}}+10000 \frac{k_{\mathrm{a}}^{2} p_{\mathrm{e}}}{\sigma_{\mathrm{c}, 0}^{2}}
$$

dove: $\sigma_{\mathrm{c}, 0}$ è la resistenza media a compressione, $k_{\mathrm{a}}$ è un parametro dipendente dalla resistenza a trazione e dalla percentuale volumetrica delle staffe e $p_{\mathrm{e}}$ è la pressione laterale effettiva esercitata sul calcestruzzo (vedere [19] per 
approfondimenti). L'energia di frantumazione per il calcestruzzo non confinato, $G_{\mathrm{F}, 0}^{\mathrm{c}}$, può essere valutata mediante le seguente espressione:

$$
G_{\mathrm{F}, 0}^{\mathrm{c}}=80-50 k_{\mathrm{b}}
$$

dove il parametro $k_{\mathrm{b}}$ dipende dalla resistenza a compressione del calcestruzzo.

Variando la classe di resistenza del calcestruzzo tra 20 e $90 \mathrm{MPa}$, l'Eq. (4) dà un'energia variabile tra 30 e $58 \mathrm{~N} / \mathrm{mm}$. Si può notare come l'energia di crushing sia tra due e tre ordini di grandezza superiore all'energia di frattura, mentre il valore critico della compenetrazione, $w_{\mathrm{cr}} \approx 1 \mathrm{~mm}$, è di un ordine di grandezza superiore al valore critico di apertura della fessura (si vedano anche i risultati delle prove sperimentali di Jansen e Shah [18]). Infine, si può evidenziare che, nel caso di calcestruzzo compresso confinato, l'energia di crushing calcolata con l'Eq. (3) e il corrispondente valore critico della compenetrazione, aumentano considerevolmente.

Il modello proposto, basato su una compenetrazione fittizia, permette di ottenere la legge costitutiva effettiva del materiale, indipendente dalla dimensione strutturale. La validità di questo approccio può essere dimostrata con provini caratterizzati non solo da snellezze differenti $[17,18]$ ma anche da dimensioni diverse. A tal proposito, si considerino le prove sperimentali condotte da Ferrara e Gobbi [26] su provini in calcestruzzo con tre differenti snellezze $(0.5,1.0$ e 2.0$)$ e tre differenti scale variabili nel rapporto 1:2:4. Le curve tensione adimensionalizzata in funzione della deformazione media ottenute sperimentalmente sono riportate in Fig. 6a, ove con S si indicano i provini aventi sezione trasversale pari a 50x50 $\mathrm{mm}$, con M 100x100 mm ed L 150x150 mm. La buona sovrapposizione dei rami crescenti di dette curve indica che, come ci si poteva aspettare, la fase elastica è indipendente da qualsiasi parametro geometrico e la sua pendenza è definita dal modulo elastico. Al contrario, i rami di post-picco sono altamente influenzati dalla snellezza e dalla scala del provino. Ciò comporta che la legge tensione-deformazione non possa essere assunta come caratteristica del materiale.

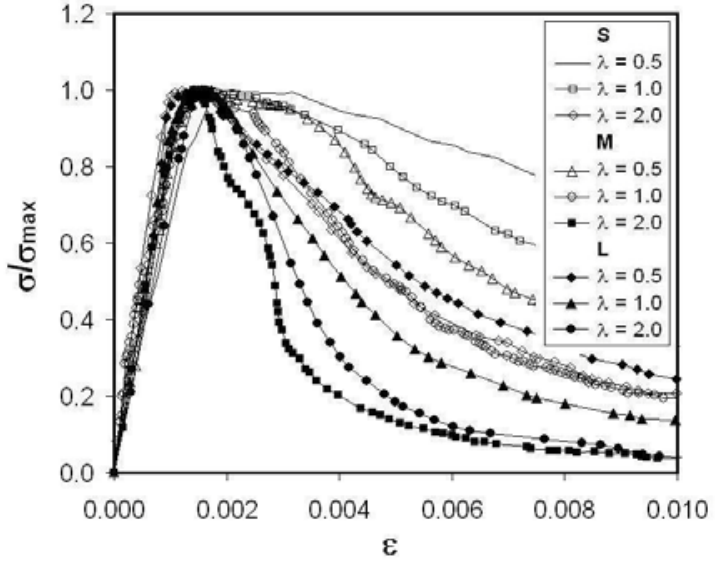

(a) legami $\sigma-\varepsilon$

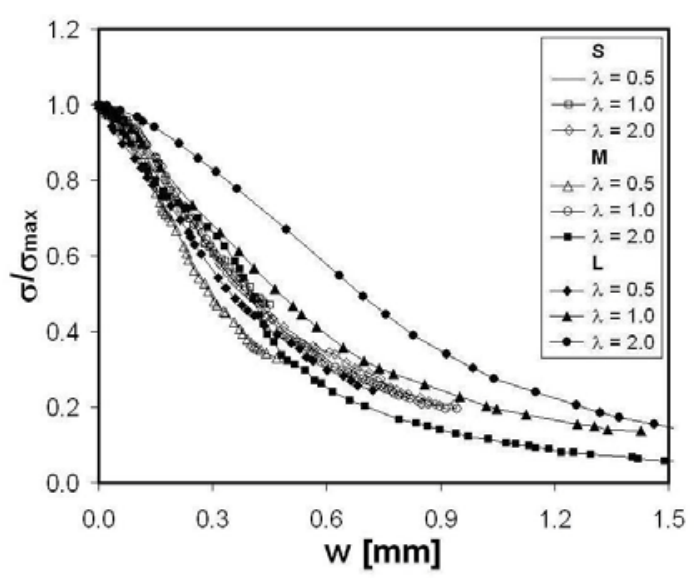

(b) legami post-picco $\sigma-w$

Figura 6: Test a compressione condotti da Ferrara e Gobbi [26]:

legami tensione-deformazione (a); legge di overlapping (b).

Considerazioni totalmente differenti possono invece essere svolte con riferimento alle leggi di overlapping. I legami $\sigma-w$ relativi alla fase di post-picco vengono ricavati dalle curve $\sigma-\varepsilon$ secondo la seguente procedura:

1) Si valuta la legge tensione-schiacciamento totale del provino $(\sigma-\delta)$ moltiplicando le deformazioni $\varepsilon$ per l'altezza del provino;

2) Dalle curve $\sigma-\delta$ ottenute si sottrae l'espansione elastica del provino, $\delta_{\mathrm{el}}$, dovuta allo scarico tensionale, e la componente plastica della fase pre-picco, $\delta_{\mathrm{p}}$, così come rappresentato in Fig. 7 . Lo spostamento $\delta_{\text {el }}$ è valutato mediante la seguente espressione:

$$
\delta_{\mathrm{el}}=\frac{\sigma_{c}}{E_{\tan }} L
$$

dove: $\sigma_{\mathrm{c}}$ è la tensione agente, $E_{\tan }$ è il modulo elastico tangente ed $L$ è l'altezza totale del provino. 
Le relazioni $\sigma-w$ determinate per le prove sperimentali di Ferrara e Gobbi sono riportate in Fig. 6b. Come si può vedere, le curve rappresentative del comportamento di softening collassano in una banda molto ristretta, confermando la scarsa dipendenza della legge di overlapping dalla snellezza e dalla scala del provino.

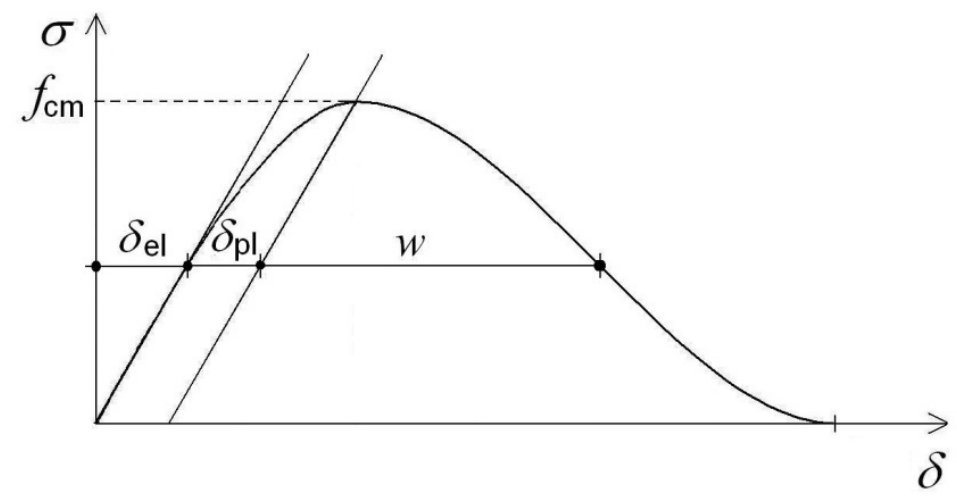

Figura 7: Procedura per ottenere la legge di overlapping a partire dal legame tensione-schiacciamento totale, $\delta$.

\section{Legge costitutiva dell'armatura}

Nella pratica corrente, le leggi costitutive maggiormente utilizzate per descrivere il comportamento dell'acciaio sono definite in campo $\sigma-\varepsilon$, quali, ad esempio, quella elasto-plastica e quella elasto-incrudente. Nel nuovo modello che viene qui proposto, non è possibile utilizzare siffatte relazioni in quanto la sezione di mezzeria del concio analizzato è cinematicamente descritta mediante spostamenti anziché deformazioni. Sarà pertanto necessario introdurre una legge che leghi la tensione agente nell'armatura con l'apertura della fessura in corrispondenza del rinforzo stesso. In passato tale problema è stato risolto in modo semplice ma efficace imponendo un comportamento di tipo rigido-plastico, cioè imponendo che l'apertura della fessura sia nulla fino a che l'armatura non si snervi [27]. Nel presente modello si vuole invece adottare un approccio più vicino alla realtà, nella quale l'apertura della fessura è ammessa anche in assenza di snervamento dell'acciaio. Ciò è reso possibile dagli scorrimenti relativi tra calcestruzzo e armatura.

Le tipiche relazioni tra scorrimento e aderenza sono definite in termini di tensioni tangenziali tra acciaio e calcestruzzo in funzione degli spostamenti relativi tra i due materiali [2]. L'integrazione degli scorrimenti lungo la lunghezza di trasferimento è pari a metà dell'apertura della fessura in corrispondenza del rinforzo. D'altra parte, l'integrazione delle tensioni tangenziali è pari alla reazione dell'armatura. Al fine di semplificare i calcoli, è stata assunta una legge lineare fino al raggiungimento dello snervamento dell'acciaio, e costante successivamente. Il parametro caratterizzante tale relazione è pertanto l'apertura corrispondente allo snervamento, $w_{y}$, che è stata assunta pari a $0.3 \mathrm{~mm}$.

\section{Algoritmo numerico}

Nel modello numerico il concio di trave da analizzare è considerato come costituito da due parti simmetriche aventi comportamento perfettamente elastico e connesse tra loro mediante $n$ coppie di nodi lungo la sezione di mezzeria, come rappresentato in Fig. 8. In corrispondenza di tali nodi agiranno le forze nodali equivalenti coesive e di overlapping e la forza di richiusura esercitata dall'armatura. Tutte queste forze dipendono dagli spostamenti nodali di apertura della fessura e di sovrapposizione nella zona di crushing, secondo le leggi costitutive introdotte nei precedenti paragrafi. Così facendo, nella sezione di mezzeria saranno concentrati i contributi di non-linearità.

Con riferimento alla Fig. 8, le forze nodali orizzontali agenti sulla sezione di mezzeria sono date dalla seguente espressione:

$$
\{F\}=\left[K_{w}\right]\{w\}+\left\{K_{M}\right\} M
$$

dove: $\{F\}$ è il vettore delle forze nodali, $\left[K_{m}\right]$ è la matrice dei coefficienti di influenza per gli spostamenti nodali, $\{w\}$ è il vettore degli spostamenti nodali, $\left\{K_{M}\right\}$ è il vettore dei coefficienti di influenza per il momento applicato ed $M$ è il momento applicato. I coefficienti di influenza, $K_{w}^{\mathrm{ij}}$, presentano la dimensione fisica di una rigidezza e sono calcolati a priori con un'analisi agli elementi finiti, imponendo spostamenti unitari a ciascuno degli $n$ nodi in Fig. 8. Nella generica situazione che si verifica durante il processo di carico, rappresentata in Fig. 9, si considerano le seguenti equazioni:

$$
F_{\mathrm{i}}=0 \quad \text { per } \mathrm{i}=1,2, \ldots,(j-1) ; \mathrm{i} \neq \mathrm{r}
$$




$$
\begin{aligned}
& F_{\mathrm{i}}=F_{\mathrm{t}, \mathrm{u}}\left(1-\frac{w_{\mathrm{i}}^{\mathrm{t}}}{w_{\mathrm{c}}^{\mathrm{t}}}\right) ; \quad \text { per } \mathrm{i}=j, \ldots,(m-1) ; \mathrm{i} \neq r \\
& w_{\mathrm{i}}^{\mathrm{t}}=0 ; \quad \text { per } \mathrm{i}=m, \ldots, p \\
& F_{\mathrm{i}}=F_{\mathrm{c}, \mathrm{u}}\left(1-\frac{w_{\mathrm{i}}^{\mathrm{c}}}{w_{\mathrm{c}}^{\mathrm{c}}}\right) ; \quad \text { per } \mathrm{i}=(p+1), \ldots, n \\
& F_{\mathrm{r}}=f\left(w_{\mathrm{r}}\right) ; \text { per } \mathrm{i}=r
\end{aligned}
$$

L'Equazione (7e) rappresenta la legge costitutiva dell'armatura.

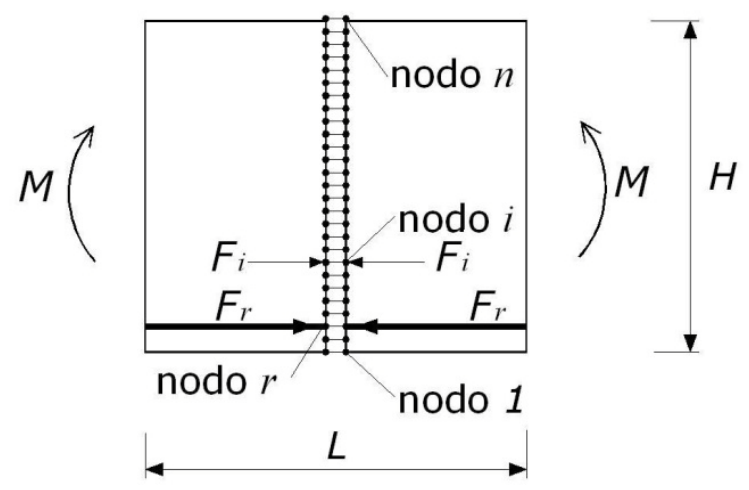

Figura 8: Discretizzazione della sezione di mezzeria mediante $n$ nodi.

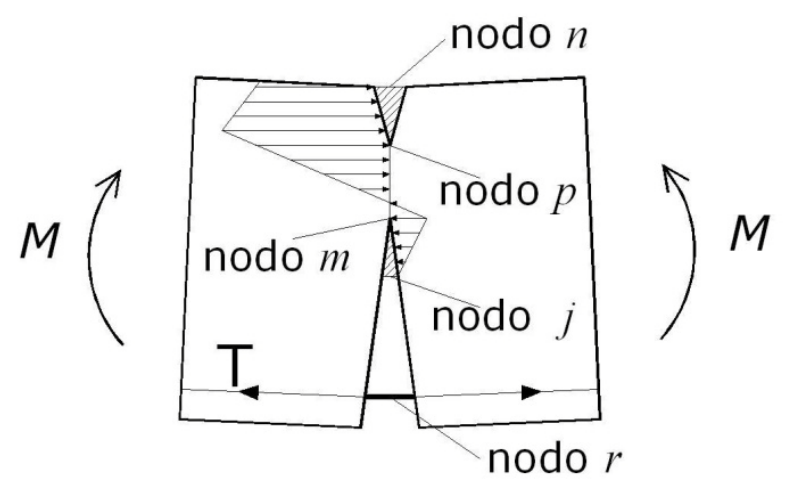

Figura 9: Distribuzione delle forze nodali con fessura coesiva in trazione e crushing in compressione.

Le Equazioni (6) e (7) costituiscono un sistema algebrico lineare di $2 n$ equazioni in $2 n+1$ incognite, cioè $\{F\},\{w\}$ ed $M$. L'ulteriore equazione necessaria per la soluzione si ottiene imponendo che la forza agente nell'apice della fessura fittizia raggiunga la resistenza a trazione del materiale o che la forza agente nell'apice dell'overlapping fittizio raggiunga la resistenza a compressione. Naturalmente, tra queste due condizioni, si impone quella più prossima alla criticità. Il parametro guida è la posizione dell'apice che nel passo di soluzione considerato ha raggiunto la crisi. Tale apice viene fatto avanzare di una posizione al passo successivo.

Infine, ad ogni passo di soluzione, è possibile calcolare la rotazione totale del concio, valutata in corrispondenza delle facce libere, ove è applicato il momento flettente, mediante la seguente relazione:

$$
\vartheta=\left\{D_{w}\right\}^{\mathrm{T}}\{w\}+D_{M} M
$$

dove: $\left\{D_{w}\right\}$ è il vettore dei coefficienti di influenza per gli spostamenti nodali, e $D_{M}$ è il coefficiente di influenza per il momento applicato. 


\section{CONFRONTO CON I RISULTATI SPERIMENTALI E PROPOSTA PER LA NORMATIVA}

$\mathrm{I}$ n questo paragrafo si propone un confronto tra i risultati numerici ottenuti mediante il modello coesivo/overlapping e quelli delle prove sperimentali condotte da Bosco e Debernardi [14]. In [14] sono state testate con lo schema di flessione su tre punti travi aventi snellezza constante, pari a 10, e differente altezza, pari a 200, 400 e $600 \mathrm{~mm}$. Sono state considerate differenti percentuali di armatura tesa, variabili tra $0.12 \%$ e $1.71 \%$. I risultati delle simulazioni numeriche sono confrontati con le rotazioni dei conci posti a cavallo della mezzeria delle travi testate, caratterizzati da una lunghezza pari all'altezza.

Le curve momento-rotazione, sia numeriche che sperimentali, sono riportate nelle Fig. 10a-c, per differenti altezze e percentuali di armatura tesa. Tali diagrammi mostrano come la rotazione massima sia una funzione decrescente della quantità di armatura e della dimensione della trave. Nel caso di bassa percentuale di armatura, il comportamento meccanico è caratterizzato dalla snervamento dell'acciaio così che la risposta strutturale è per lo più plastica. Incrementando la quantità di armatura, il crushing del calcestruzzo diventa via via più evidente con la comparsa di un ramo di softening alla fine del tratto plastico.

Questa è una caratteristica importante del modello proposto, che permette di seguire rami instabili caratterizzati da pendenza positiva (snap-back). Ciò è possibile in quanto il parametro di controllo del processo di carico è l'estensione della fessura coesiva e della zona di crushing, piuttosto che il carico applicato o la freccia in mezzeria. In generale, si può notare un buon accordo tra le simulazioni numeriche e i risultati sperimentali.

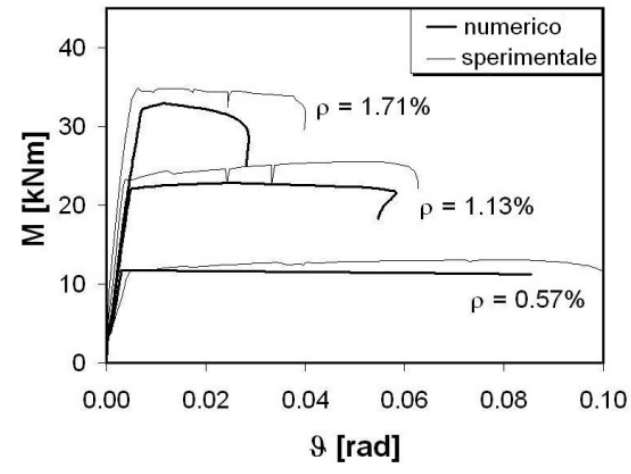

(a) $\mathrm{h}=200 \mathrm{~mm}$

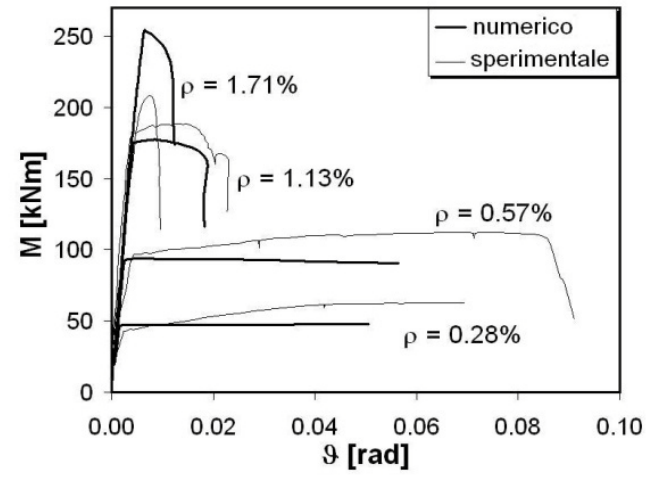

(b) $\mathrm{h}=400 \mathrm{~mm}$

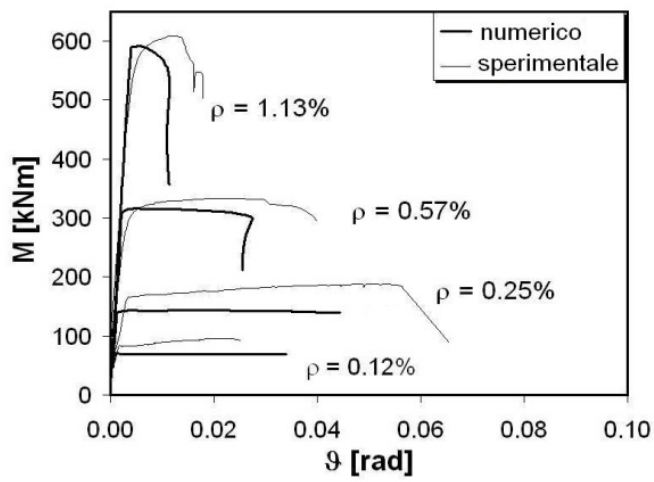

(c) $\mathrm{h}=600 \mathrm{~mm}$

Figura 10: Confronto tra risultati numerici e sperimentali per travi con altezze differenti: $h=200 \mathrm{~mm}(\mathrm{a}) ; h=400 \mathrm{~mm}(\mathrm{~b}) ; h=600 \mathrm{~mm}$ (c).

Dopo la validazione preliminare del modello, si è condotta un'analisi parametrica al fine di analizzare l'effetto della dimensione strutturale e della percentuale di armatura sulla duttilità delle travi. L'andamento tipico dei risultati ottenuti è quello riportato in Fig. 11 per una percentuale di armatura in trazione pari al $2 \%$. Il momento flettente è adimensionalizzato al fine di migliorare il confronto tra i risultati. Il plateau orizzontale è dovuto allo snervamento dell'acciaio. Le curve rappresentate evidenziano una diminuzione della rotazione massima dei conci di trave all'aumentare della dimensione della trave stessa, con la comparsa di rami di softening con una pendenza via via crescente. 


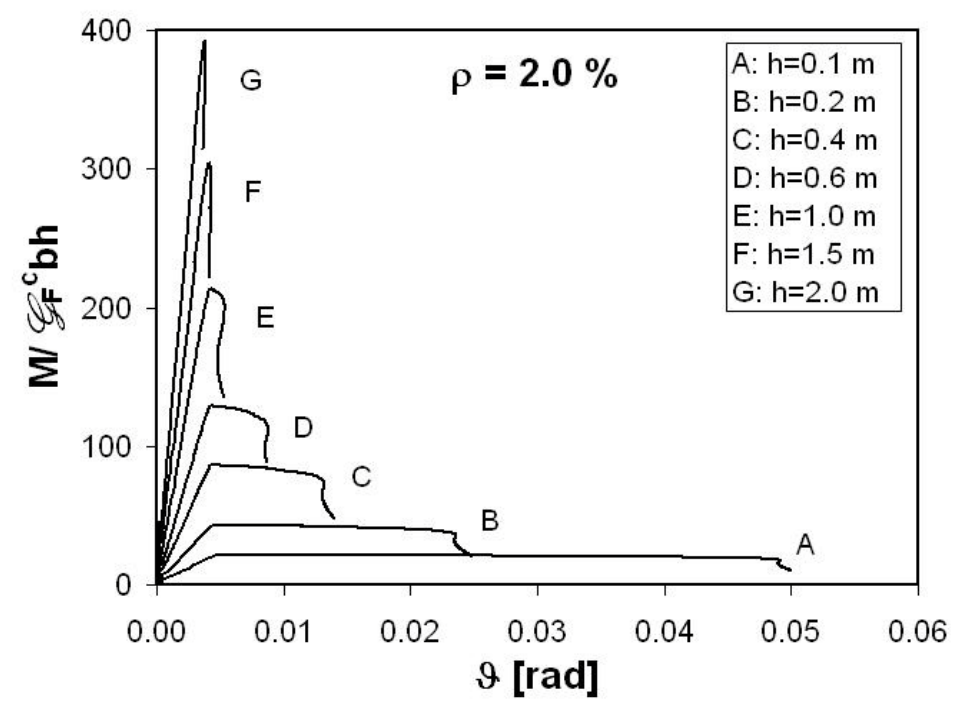

Figura 11: Momento flettente adimensionalizzato in funzione della rotazione totale del concio per $\rho_{\mathrm{t}}=2 \%$ e differenti altezze.

Una caratteristica del presente modello è che non è necessario introdurre alcuna ipotesi sulle deformazioni, come ad esempio l'ipotesi di Bernoulli. Al contrario, gli spostamenti nodali della sezione di mezzeria sono ottenuti dalla procedura numerica di soluzione passo dopo passo. I profili degli spostamenti nodali relativi alla trave con altezza pari a $0.4 \mathrm{~m}$ e percentuale di armatura pari a 2\% sono riportati in Fig. 12 per differenti valori di carico. Per bassi valori del momento flettente, fino a circa il $60 \%$ del momento di snervamento, il comportamento meccanico è caratterizzato da un progressivo avanzamento e apertura della fessura in trazione. Il danneggiamento del calcestruzzo in compressione comincia appena prima dello snervamento dell'acciaio e cresce all'avvicinarsi del momento ultimo.
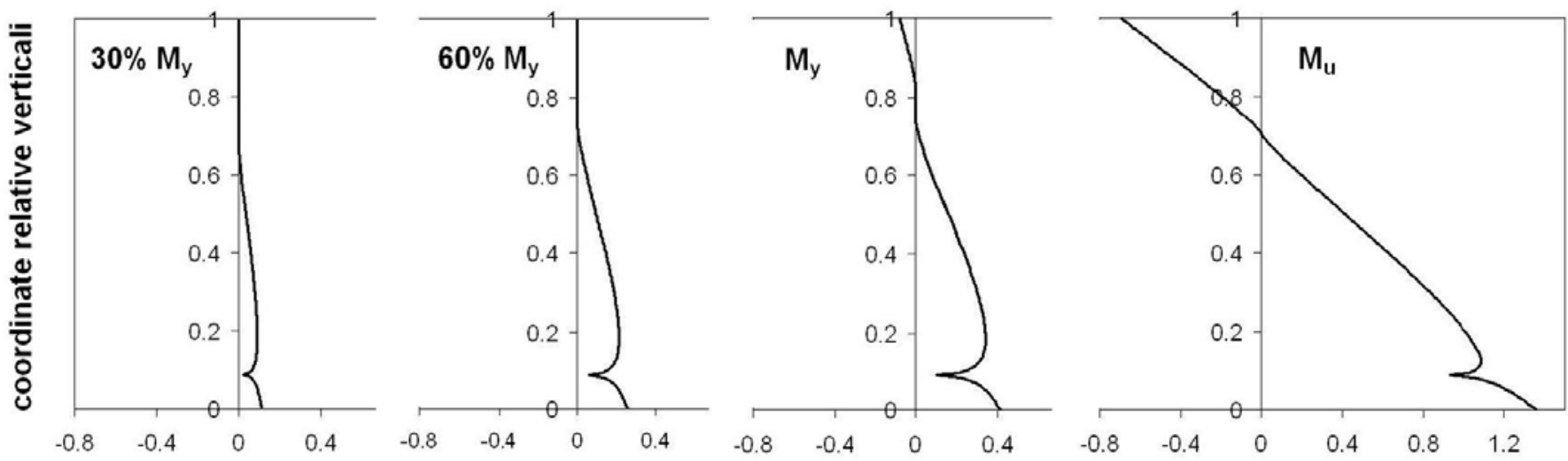

\section{spostamenti nodali [mm]}

Figura 12: Spostamenti nodali della sezione di mezzeria del concio per differenti livelli di carico, con $b=400 \mathrm{~mm} \mathrm{e} \rho_{\mathrm{t}}=2 \%$.

Con riferimento ai diagrammi momento-rotazione, è possibile valutare la capacità di rotazione plastica come differenza tra la rotazione ultima e quella relativa allo snervamento dell'acciaio. In particolare, nel presente lavoro si è scelta la definizione di rotazione ultima data da Hillerborg [3] e Pecce [4]. I risultati dell'analisi parametrica possono così essere riassunti nel grafico di Fig. 13, che mette in relazione la rotazione plastica con la profondità relativa dell'asse neutro, $x / d$, coerentemente con quanto previsto dalle normative. La curva tratteggiata è quella fornita dall'Eurocodice 2 per acciaio ad alta duttilità e classe di resistenza del calcestruzzo inferiore o uguale a $50 \mathrm{MPa}$, mentre le curve a tratto pieno sono relative ai risultati numerici. Travi con un'altezza pari a $0.2 \mathrm{~m}$ esibiscono una capacità rotazionale superiore a quella indicata dalla normativa, mentre travi con altezza pari a $0.6 \mathrm{~m}$ o $0.8 \mathrm{~m}$ hanno una capacità di rotazione inferiore. Ciò evidenzia una forte influenza della dimensione strutturale, completamente ignorata dalle normative. 


\section{CONCLUSIONI}

$\mathrm{N}$

el presente lavoro, si è proposto un algoritmo numerico per l'analisi del comportamento duttile di elementi in calcestruzzo armato in flessione. Al fine di ottenere un'accurata valutazione del diagramma momento-rotazione, si sono considerati i principali contributi non-lineari del calcestruzzo e dell'armatura. Dalle simulazioni numeriche si traggono le seguenti conclusioni:

1) La nuova legge costitutiva per il calcestruzzo in compressione, denominata Overlapping Crack Law [25], permette di descrivere il comportamento non-lineare considerando gli effetti della scala e di cogliere i rami di softening presenti al termine dei diagrammi momento rotazione, come mostrato in Fig. 11.

2) Con riferimento alla Fig. 10, è possibile affermare che l'algoritmo proposto coglie i risultati sperimentali [14] al variare della dimensione strutturale e della percentuale di armatura.

3) Indipendentemente dalla percentuale di armatura, il comportamento diventa più fragile all'aumentare della dimensione della trave, con una progressiva riduzione della rotazione ultima.

4) L'Eurocodice 2 [11] esprime la rotazione plastica ammissibile di travi in calcestruzzo armato come funzione solamente della posizione dell'asse neutro. Al fine di migliorare tali prescrizioni, l'effetto della scala dovrebbe essere introdotto in modo esplicito, considerando differenti curve di progetto, come ad esempio mostrato in Fig. 13.

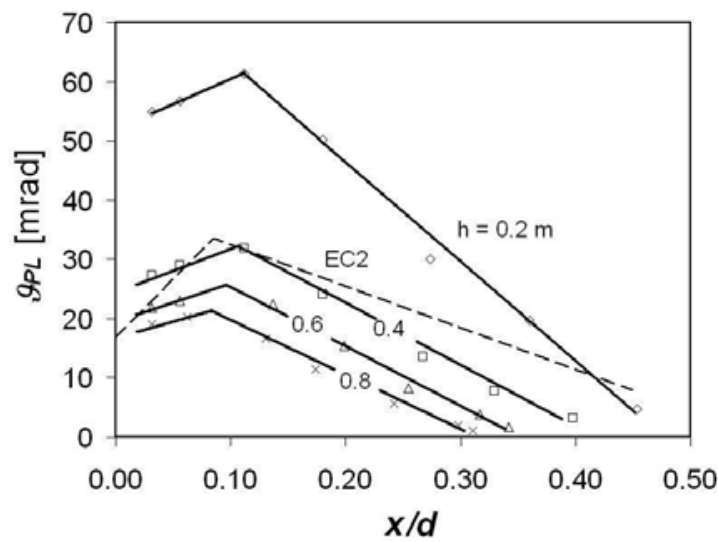

Figura 13: Rotazioni plastiche ottenute con il modello proposto confrontate con le prescrizioni dell'EC2.

\section{BIBLIOGRAFIA}

[1] G. Macchi, Costruzioni in Cemento Armato, Studi e Rendiconti, 6 (1969) 151-191.

[2] Comite Euro-International du Beton, CEB-FIP Model Code 1990, Thomas Telford Ltd, Lausanne, Bulletin No. 213/214 (1993).

[3] A. Hillerborg, Engineering Fracture Mechanics, 35 (1990) 233-240.

[4] M. Pecce, CEB Bulletin d'Information No. 242 (1997) 197-210.

[5] CEB, Bulletin d'Information No. 30, 1961.

[6] A.L.L. Baker, A.M.N. Amakarone, Proceedings of Conference on Flexural Mechanics of Reinforced Concrete, Special Publication SP12. American Concrete Institute (1967).

[7] R. Eligehausen, P. Langer, CEB Bulletin d'Information No. 175 (1987) I 7.9-I 7.27.

[8] E. Cosenza, C. Greco, G. Manfredi, Atti dell'Accademia Nazionale dei Lincei, IX(2) (1991) 249-258.

[9] N. Tue, L. Qian, D. Pommerening, Technische Hochschule Darmstadt, Az. IV 1-5 (1996) 683-692.

[10] A.J. Bigaj, J. Walraven, Heron, 47 (2002) 53-75.

[11] EN 1992-1-1, Eurocode 2: Design of concrete structures - Part 1-1: General rules and rules for buildings, 2003.

[12] G.W. Corley, Journal of Structural Division, 92 (1966) 121-146.

[13] E. Siviero, CEB Bulletin d'Information No. 105 (1974) 206-222.

[14] C. Bosco, P.G. Debernardi, Report No. 36, Atti del Dipartimento, Politecnico di Torino, Ingegneria Strutturale, (1992).

[15] A.J. Bigaj, J. Walraven, CEB Bulletin d'Information No. 218 (1993) 7-23.

[16] Z.P. Bažant, Cement Concrete Research, 19 (1989) 973-977. 
[17] M. van Vliet, J. van Mier, Mechanics of Cohesive-Frictional Materials, 1 (1996) 115-127.

[18] D.C. Jansen, S.P. Shah, Journal of Engineering Mechanics, 123 (1997) 25-35.

[19] M. Suzuki, M. Akiyama, H. Matsuzaki, T.H. Dang, Procedings of the 2nd fib International Conference, Napoli, Italy, 2006, CD-ROM, ID 3-13 (2006).

[20] H. Dahl, R. Brincker, Proceedings of the International Conference on Recent Developments in the Fracture of Concrete and Rock, Cardiff, Wales, 1989, Elsevier Applied Science, England, (1989) 523-536.

[21] A. Carpinteri, G. Lacidogna, N. Pugno, International Journal of Fracture, 129 (2004) 131-139.

[22] G. Ferro, A. Carpinteri, Journal of Applied Mechanics, 75 (2008) 41003/1-41003/8.

[23] A. Carpinteri, Proceedings of a NATO Advanced Research Workshop, Evanston, USA, 1984, Martinus Nijhoff Publishers, Dordrecht, (1985) 287-316.

[24] A. Carpinteri, Journal of the Mechanics and Physics of Solids, 37 (1989) 567-582.

[25] A. Carpinteri, M. Corrado, M. Paggi, G. Mancini, Proceedings of FraMCoS 6, Catania, Italy, 2007, Taylor \& Francis, London, 2 (2007) 655-663.

[26] G. Ferrara, M.E. Gobbi, Report to RILEM Committee 148-SSC, ENEL-CRIS Laboratory, Milano, (1995).

[27] C. Bosco, A. Carpinteri, Journal of Engineering Mechanics (ASCE), 118 (1991) 1564-1577. 